\title{
Caracterização espectroscópica de cristais de ácidos láurico e mirístico
}

\author{
Spectroscopic characterization of lauric and myristic acid crystals \\ L.G. Melo ${ }^{1}$, A.P.S. Oliveira ${ }^{2 *}$; K.H.G. Freitas ${ }^{3}$; F.F. de Sousa ${ }^{1}$ \\ ${ }^{1}$ Instituto de Ciencias Exatas, Universidade Federal do Sul e Sudeste do Pará, 68505-080, Marabá-Pará, Brasil, \\ ${ }^{2}$ Programa de Pós Graduação em Química da Universidade Federal do Ceará, 60455-760, Fortaleza-Ceará, Brasil \\ ${ }^{3}$ Faculdade de Engenharia de Materiais, Campus de Ananideua, Universidade Federal do Pará, 66075-900, \\ Ananideua-Pará, Brasil \\ *oliveirasapaula@gmail.com
}

(Recebido em 09 de setembro de 2016; aceito em 25 de novembro de 2016)

\begin{abstract}
Resumo:
Os ácidos láurico e mirístico são componentes de diversos óleos e gorduras vegetais de interesse industrial. Mesmo em pequena quantidade, eles exercem boa influência nas propriedades físicas e químicas de óleos vegetais a que pertencem. O conhecimento das suas propriedades espectroscópicas fornecem informações importantes a respeito de sua estrutura molecular, inclusive da rede cristalina para o caso de cristais, informações que contribuem para o entendimento do seu comportamento quando submetido à condições extremas de temperatura e pressão, e pode dar suporte à aplicações em diversas engenharias, como é o caso da engenharia de alimentos e engenharia química. Neste trabalho realizou-se a caracterização espectroscópica dos cristais de ácido láurico e mirístico na forma $\mathrm{C}$ (monoclínico) através do espalhamento Raman não polarizado. Todos os modos de vibração da rede e internos da molécula foram identificados e classificados baseando-se em dados de outros ácidos graxos de mesma natureza, neste caso, ácidos graxos monocarboxilados.
\end{abstract}

Palavras-chave: Cristais de ácidos láurico e mirístico, Modos vibracionais, Espalhamento Raman

Abstract:

The lauric and myristic acids are components of many vegetable oils and fats of Amazon with wide application in industry. They have large influence on physical and chemical properties of vegetable oils, even in small quantity. The knowledge of their spectroscopic properties provides important information about its molecular structure, including the crystal lattice. Such information contributes to the understanding of its behavior when subjected to extreme conditions of temperature and pressure, which support the applications in various areas, such as food engineering and chemical engineering. In this work, it was made spectroscopic characterization of lauric and myristic acid crystals in the $\mathrm{C}$ form (monoclinic) by non-polarized Raman scattering. All the vibrational modes of lattice and internal of the molecule were identified and classified on data of other monocarboxylic fatty acids already published.

Keywords: Lauric and myristic acid crystals, Vibrational modes, Raman scattering

\section{INTRODUÇÃO}

Ácidos graxos possuem papel importante nos campos da indústria de alimentos, de cosméticos, farmacêutica e na medicina. Eles desempenham também papel fundamental no organismo humano por serem fontes de energia para o corpo. Os ácidos graxos são importantes tanto presentes em óleos quanto isolados [1]. Eles podem ser definidos como sistemas moleculares que possuem cadeias curta, média e longa, podendo ser classificados em saturados e insaturados, essenciais e não essenciais. A presença de insaturações nos ácidos graxos atribuem à eles diferentes propriedades físico-químicas [2], por isso, possuem propriedades peculiares, potencializando-os para a utilização nas mais diversas áreas do conhecimento [2].

Os ácidos mirístico e láurico são ácidos de cadeia carbônica saturada que podem ser encontrados em óleos vegetais Amazônicos, como os óleos de palmiste, babaçu e macaúba. O 
ácido láurico $\left(\mathrm{C}_{12} \mathrm{H}_{24} \mathrm{O}_{2}\right)$ possui ação antimicrobiana e antibactericida [3]. Ele também atribui aos óleos láuricos (óleos com uma quantidade considerável de ácido láurico em sua composição) excelentes características para a produção de biodiesel [4]. O ácido mirístico $\left(\mathrm{C}_{14} \mathrm{H}_{28} \mathrm{O}_{2}\right)$ junto com ácido láurico está entre os ácidos graxos mais abundantes na natureza, presente também na gordura do coco, leite e na maioria dos óleos e gorduras de origem vegetal e animal [1].

O conhecimento das propriedades físicas e químicas de ácidos graxos contribui para um melhor aproveitamento dos óleos e gorduras a que pertencem. Informações a respeito das propriedades de materiais como os ácidos graxos podem ser obtidas de maneira precisa por meio de técnicas espectroscópicas. A espectroscopia Raman é uma técnica experimental muito utilizada na caracterização de materiais e vem como uma ferramenta útil no esclarecimento das propriedades vibracionais de cristais de ácidos graxos [5].

Considerando-se a falta de informação a respeito das propriedades físicas e químicas dos ácidos láurico e mirístico, desenvolveu-se este trabalho voltado à caracterização espectroscópica dos cristais simples de ácidos láurico e mirístico ambos na forma $\mathrm{C}$ (monoclínica) em condições ambiente por meio do espalhamento Raman (espectroscopia Raman) não polarizado.

\section{MATERIAL E MÉTODOS}

\subsection{Amostras}

As amostras de ácidos láurico e mirístico foram adquiridas no mercado por meio da empresa comercial Merck (U.S.A.) com nível de pureza maior ou igual a 99\%. Para obter a fase polimórfica (forma $\mathrm{C}$ ) estudada neste trabalho, foi necessário recristalizar a amostra usando variação de temperatura até um valor acima do ponto de fusão e, em seguida, resfriada lentamente até um valor bem abaixo deste, obtendo assim a fase desejada.

\subsection{Medidas de difração de raios $X$}

Para tais medidas, a amostra em pó foi fixada a um porta amostra de silício por colagem com pasta de silicone e submetidas à incidência de um feixe de raios-X. As condições de

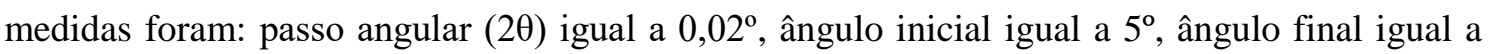
$50^{\circ}$, e velocidade angular do feixe $(2 \theta / \mathrm{min})$ igual a 0,5 para todas os dois cristais, no modo contínuo. O equipamento utilizado foi um difratômetro Rigaku (DMAXB) configurado numa geometria Bragg-Bretano, operando em $40 \mathrm{kV} / 25 \mathrm{~mA}$ com um tubo de $\mathrm{Cu}-\mathrm{K} \alpha$ e usando um monocromador de grafite.

\subsection{Medidas de espectros Raman}

Os espectros Raman dos ácidos láurico e mirístico obtidos neste trabalho foram medidos com a utilização de um sistema micro-Raman usando a geometria retro-espalhamento (backscattering) com um laser de Argônio da Coherent modelo 70c emitindo na linha 514,5 nm. Também foi utilizado um espectrômetro triplo da Jobin-Yvon modelo T 64000 e um sistema detetor CCD (Charge-Coupled Device) resfriado a nitrogênio líquido. As fendas foram ajustadas de modo a obter medidas espectrais com resolução de aproximadamente $2 \mathrm{~cm}^{-1}$. Para focar o laser na amostra foi utilizado um microscópico da marca Olympus modelo BX40, que é 
acoplado a uma câmara de vídeo, com uma lente de aumento de 20x e distância focal de 20,5 $\mathrm{mm}$.

\section{RESULTADOS E DISCUSSÃO}

\subsection{Caracterização estrutural dos ácidos Láurico e Mirístico}

O padrão de difração foi medido na região angular entre 5 e $50^{\circ}$ (em 20). Com ajuda da literatura [6], verificou-se que os dois ácidos encontram-se na conformação C, cuja simetria é monoclínica com grupo espacial $P 2_{1} / c\left(C_{2 h}^{5}\right)$ com quatro moléculas por célula unitária $(\mathrm{Z}=4)$. De acordo com a literatura [6] os valores dos parâmetros de rede são aqueles que estão apresentados logo a seguir na Tabela 1 .

Tabela 1: Parâmetros de rede dos cristais de ácidos láurico e mirístico.

\begin{tabular}{llll}
\hline \hline Ácido (forma C) & $\boldsymbol{a}^{*}$ & $\boldsymbol{b}$ & $\boldsymbol{c}$ \\
\hline Láurico & 9,604 & 4,953 & 42,30 \\
Mirístico & 9,497 & 4,972 & 44,45 \\
\hline \hline
\end{tabular}

*Unidades em Angstrom. Fonte: Adaptado da ref. [6].

\subsection{Propriedades vibracionais dos ácidos Láurico e Mirístico}

Na Figura 1, são apresentados os espectros Raman dos cristais de ácidos láurico e mirístico (forma C) na região espectral de 40 até $3000 \mathrm{~cm}^{-1}$. As propriedades vibracionais dos dois cristais são discutidas logo a seguir. Todos os modos Raman ativos foram identificados e classificados com a ajuda de resultados previamente publicados de ácidos graxos ou moléculas de estruturas semelhantes. Geralmente em ácidos graxos, os modos de vibração da rede cristalina (ou modos externos) são observados na região entre 10 e $180 \mathrm{~cm}^{-1}$ e os modos internos da molécula estão contidos na faixa espectral $180-3100 \mathrm{~cm}^{-1}$. É conhecido na literatura que alguns desses modos possuem acoplamentos com modos internos [7]. A segunda região espectral contêm modos normais de vibração específicos da própria molécula que estão associados às vibrações do tipo: stretching (estiramento) que pode ser simétrico ou assimétrico, scissoring (corte), wagging (balanço em fase), twisting (balanço fora de fase), rocking (oscilação) e torções [7-10].

As propriedades dos cristais de ácidos mirístico e láurico serão discutidas com detalhes a seguir. Os modos associados à vibrações da rede, que são também conhecidos como vibrações intermoleculares, foram observados em 53, 68, 78 e $99 \mathrm{~cm}^{-1}$ para o ácido láurico e em 161 e 167 $\mathrm{cm}^{-1}$ para o ácido mirístico [10]. Foram observados em 183, 187 e $207 \mathrm{~cm}^{-1}$ modos associados a torções de ligações entre carbonos, $\tau\left(\right.$ CCCC), para o ácido mirístico, e em $206 \mathrm{~cm}^{-1}$ foi observado um modo para o ácido láurico, que foi associado ao mesmo tipo de vibração [7]. Os modos na região de 400 até $655 \mathrm{~cm}^{-1}$ foram classificados como deformações das ligações entre carbonos, $\delta(\mathrm{CCC})[7,9,10]$. Dois modos relacionados à deformações da ligação $\mathrm{COO}^{-}$foram observados em 670 e $894 \mathrm{~cm}^{-1}$ para os ácidos mirístico e láurico [8,10]. Modos localizados em 910 e $988 \mathrm{~cm}^{-1}$ no ácido mirístico e em $909 \mathrm{~cm}^{-1}$ no ácido láurico são devido à deformações do tipo rocking do grupo $\mathrm{CH}_{2}$ [9]. A região espectral entre 1000 e $1130 \mathrm{~cm}^{-1}$, para os dois ácidos graxos, contém estiramentos da ligação entre dois carbonos $(v(C C))[7,8,10]$. Todos estes modos de vibrações e suas respectivas classificações estão apresentados na Tabela 2. 


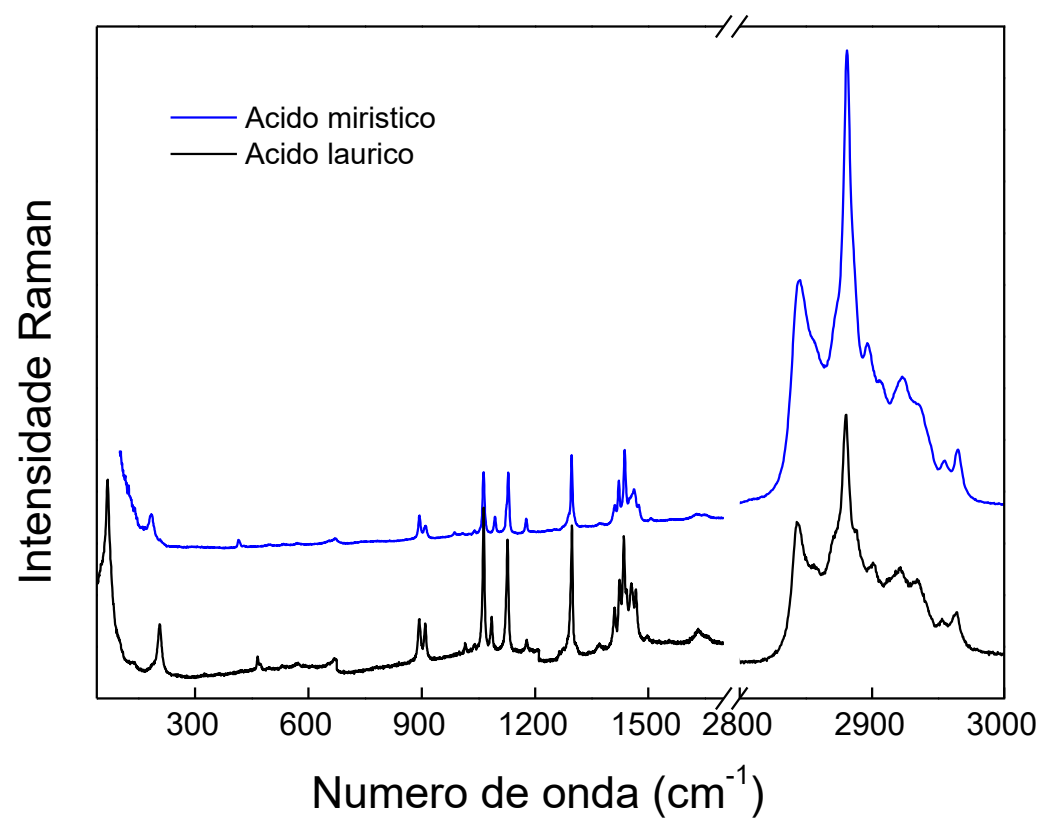

Figura 1: Espectros Raman dos ácidos mirístico e láurico na região de 20 a $3000 \mathrm{~cm}^{-1}$.

Os modos observados entre $1177 \mathrm{a} 1500 \mathrm{~cm}^{-1}$ foram associados às deformações do tipo: twisting, scissoring, wagging e rocking das unidades $\mathrm{CH}_{2}$ e $\mathrm{CH}_{3}$ (veja Tabela 2). Os modos localizados em torno de 1177 e 1297, observados nos espectros dos ácidos mirístico e láurico foram designados como a uma deformação do tipo twisting do grupo $\mathrm{CH}_{2}$ [4]. Outro modo observado no espectro do ácido láurico em $1370 \mathrm{~cm}^{-1}$ foi associado a uma deformação do tipo wagging do $\mathrm{CH}_{3}$ [9]. Os modos na região de 1420 a $1500 \mathrm{~cm}^{-1}$ foram associados a deformações do tipo scissoring do $\mathrm{CH}_{2}$ ou $\mathrm{CH}_{3}$, estes foram encontrados para os ácidos mirístico e láurico, respectivamente, em: $1422,1437,1460 \mathrm{~cm}^{-1}$ e 1424, 1435, 1442, 1455, 1467, $1497 \mathrm{~cm}^{-1}$ [4]. 
Tabela 2: Modos vibracionais Raman ativos e suas respectivas designações para os cristais de ácidos

Mirístico e Láurico

\begin{tabular}{|c|c|c|c|c|c|c|c|}
\hline $\begin{array}{c}\text { Ácido } \\
\text { Mirístic }\end{array}$ & $\begin{array}{c}\text { Ácido } \\
\text { láurico }\end{array}$ & Literatura & Designação & $\begin{array}{c}\text { Ácido } \\
\text { mirístico }\end{array}$ & $\begin{array}{c}\text { Ácido } \\
\text { láurico }\end{array}$ & Literatura & Designação \\
\hline - & 53 & $48^{10}$ & $\overline{\text { rede }}$ & 1177 & 1177 & $1178^{10}$ & $\delta\left(\mathrm{CH}_{2}\right)$ tw. $-\rho$ \\
\hline- & 68 & $64^{10}$ & rede & 1297 & 1297 & $1298^{10}$ & $\delta\left(\mathrm{CH}_{2}\right) \mathrm{tw}$ \\
\hline- & 78 & $79^{10}$ & rede & - & 1370 & $1375^{9}$ & $\operatorname{wag}\left(\mathrm{CH}_{3}\right)$ \\
\hline- & 99 & $105^{10}$ & rede & 1411 & 1410 & $1410^{7}$ & $\delta\left(\mathrm{CH}_{2}\right)$ \\
\hline 161 & - & $162^{10}$ & rede & 1422 & 1424 & $1423^{10}$ & $\delta\left(\mathrm{CH}_{2 \text { ou } 3}\right)$ scis. \\
\hline 167 & - & $162^{10}$ & rede & 1437 & 1435 & $1441^{10}$ & $\delta\left(\mathrm{CH}_{2}\right.$ ou 3$)$ scis. \\
\hline 183 & - & $187^{7}$ & $\tau(\mathrm{CCCC})$ & - & 1442 & $1441^{10}$ & $\delta\left(\mathrm{CH}_{2}\right.$ ou 3$)$ scis. \\
\hline 187 & - & $187^{7}$ & $\tau(\mathrm{CCCC})$ & - & 1455 & $1456^{10}$ & $\delta\left(\mathrm{CH}_{2 \text { ou } 3}\right)$ scis. \\
\hline 207 & 206 & $203^{7}$ & $\tau(\mathrm{CCCC})$ & 1460 & - & $1456^{10}$ & $\delta\left(\mathrm{CH}_{2 \text { ou } 3}\right)$ scis. \\
\hline 416 & - & $414^{8}$ & $\delta(\mathrm{CCC})$ & - & 1467 & $1467^{10}$ & $\delta\left(\mathrm{CH}_{2}\right.$ ou 3$)$ scis. \\
\hline 429 & - & $423^{7}$ & $\delta(\mathrm{CCC})$ & - & 1497 & $1493^{10}$ & $\delta\left(\mathrm{CH}_{2}\right.$ ou 3$)$ scis. \\
\hline- & 466 & $462^{7}$ & $\delta(\mathrm{CCC})$ & 1626 & - & $1627^{8}$ & $v(\mathrm{C}=\mathrm{O})$ \\
\hline- & 473 & $474^{7}$ & $\delta(\mathrm{CCC})$ & - & 1632 & $1634^{10}$ & $v(\mathrm{C}=\mathrm{O})$ \\
\hline 496 & - & $492^{10}$ & $\delta(\mathrm{CCC})$ & 1650 & - & $1645^{7}$ & $v(\mathrm{C}=\mathrm{O})$ \\
\hline 534 & 532 & $531^{7}$ & $\delta(\mathrm{CCC})$ & - & 1655 & $1655^{8}$ & $v(\mathrm{C}=\mathrm{O})$ \\
\hline 571 & 570 & $575^{11}$ & $\delta(\mathrm{CCC})$ & 2845 & 2844 & $2846^{9}$ & $v_{\mathrm{s}}\left(\mathrm{CH}_{2}\right)$ \\
\hline 653 & - & $656^{11}$ & $\delta(\mathrm{CCC})$ & - & 2855 & $2850^{9}$ & $v_{\mathrm{s}}\left(\mathrm{CH}_{2}\right)$ \\
\hline 670 & 669 & $670^{10}$ & $\delta \mathrm{COO}$ & 2860 & - & $2862^{7}$ & Comb. \\
\hline 894 & 894 & $893^{8}$ & $\delta \mathrm{COO}$ & - & 2871 & $2875^{7}$ & $v_{\mathrm{s}}\left(\mathrm{CH}_{3}\right)$ \\
\hline 910 & 909 & $909^{8}$ & $\rho\left(\mathrm{CH}_{2}\right)$ & 2881 & 2880 & $2882^{7}$ & $v_{a}\left(C_{2}\right)$ \\
\hline 988 & - & $986^{7}$ & $\rho\left(\mathrm{CH}_{2}\right)$ & - & 2888 & $2888^{7}$ & $v_{a}\left(C_{2}\right)$ \\
\hline 1008 & - & $1010^{7}$ & $v(\mathrm{CC})$ & - & 2901 & $2900^{10}$ & $v_{s}\left(\mathrm{CH}_{3}\right)$ \\
\hline- & 1015 & $1015^{7}$ & $v(\mathrm{CC})$ & 2908 & - & $2908^{10}$ & $v\left(\mathrm{CH}_{2}\right)$ \\
\hline 1024 & - & $1030^{7}$ & $v(\mathrm{CC})$ & - & 2912 & $2908^{10}$ & $v\left(\mathrm{CH}_{2}\right)$ \\
\hline 1040 & 1040 & $1041^{7}$ & $v(\mathrm{CC})$ & 2922 & 2921 & $2922^{9}$ & $v_{\mathrm{as}}\left(\mathrm{CH}_{3}\right)$ \\
\hline 1064 & 1064 & $1066^{7}$ & $v(C C)$ & 2937 & 2935 & $2936^{7}$ & $v\left(\mathrm{CH}_{2}\right)$ \\
\hline- & 1085 & $1087^{7}$ & $v(\mathrm{CC})$ & 2955 & 2953 & $2953^{9}$ & $v_{\mathrm{as}}\left(\mathrm{CH}_{3}\right)$ \\
\hline 1094 & - & $1092^{8}$ & $v(\mathrm{CC})$ & 2965 & 2963 & $2960^{10}$ & $v_{\mathrm{as}}\left(\mathrm{CH}_{3}\right)$ \\
\hline 1129 & 1127 & $1130^{10}$ & $v(C C)$ & - & - & - & - \\
\hline
\end{tabular}

$v$, estiramento; $v_{a s}$, estiramento assimétrico; $v_{s}$, estiramento simétrico; $\tau w$, twisting; wag, wagging; $\tau$, torção; $\delta$, deformação; $\rho$, rocking; scis, scissoring; comb., combinação de movimentos

Os modos referentes ao estiramento da carbonila $(v(\mathrm{C}=\mathrm{O}))$, neste trabalho, foram observados entre 1620 a $1660 \mathrm{~cm}^{-1}$. De acordo com a literatura [2,8,4], as bandas associadas com este tipo de movimento podem aparecer em espectros Raman entre 1600 e $1750 \mathrm{~cm}^{-1}$, dependendo de como a carbonila está posicionada no sistema molecular, uma vez que a mesma depende da natureza das ligações de hidrogênio. Nos espectros dos dois ácidos graxos deste estudo, as bandas associadas à carbonila apresentaram dois picos para cada um deles. De fato, no espectro do cristal de ácido mirístico os modos associados ao estiramento da carbonila estão localizados próximos de 1626 e $1650 \mathrm{~cm}^{-1}$, enquanto que para o ácido láurico, esses modos foram encontrados por volta de 1632 e $1655 \mathrm{~cm}^{-1}$.

Os modos na região de alta frequência entre 2800 e $3000 \mathrm{~cm}^{-1}$ foram associados à vibrações de estiramentos das unidades $\mathrm{CH}, \mathrm{CH}_{2}$ e $\mathrm{CH}_{3}$, cujos modos podem ser designados como estiramento da unidade $\mathrm{CH}$, estiramento simétrico do $\mathrm{CH}_{2}$ ou $\mathrm{CH}_{3}$ e estiramento 
assimétricos das mesmas unidades da molécula [2,4,5]. Os valores dos números de onda de todos os modos observados nesta região encontram-se na Tabela 2.

\section{CONCLUSÃO}

Os cristais de ácidos mirístico e láurico foram estudados via difração de raios $\mathrm{X}$ e espectroscopia Raman não polarizado. A partir dos experimentos de difração foi possível identificar a fase polimórfica dos cristais estudados, os quais se encontram na forma $\mathrm{C}$ com simetria monoclínica e grupo espacial $P 21 / \mathrm{a}\left(C_{2 h}^{5}\right)$ com quatro moléculas por célula unitária. As bandas Raman dos cristais dos dois ácidos foram estudadas e, também, identificadas. Com auxílio da literatura referente aos dados de espectroscopia Raman de outros ácidos graxos, foi possível classificar todos os modos de vibração desde a região de baixo número de onda (baixa energia) até a região de mais alta energia. A classificação dos modos Raman ativos de ácidos graxos em condição ambiente de temperatura e pressão, é muito importante no estudo do polimorfismo destes materiais, pois fornece informações que podem ser utilizadas como ponto de partida para avaliar a estabilidade termodinâmica, neste caso, variando a temperatura e a pressão. Este tipo de estudo, também é muito útil para diversas áreas do conhecimento, por exemplo, engenharia de materiais, engenharia de alimentos, engenharia química, farmacologia, e algumas áreas de pesquisa de base, como química e física, porque a difração de raios X e a espectroscopia Raman permitem obter informações estruturais sobre um sistema biomolecular fornecendo subsídios na caracterização de materiais. 


\section{REFERÊNCIAS BIBLIOGRÁFICAS}

1. Vianni R, Braz-Filho R. Ácidos graxos naturais: importância e ocorrencia em alimentos. Química Nova. 1995 dez; 19(4): 400-407.

2. Sousa FF. Estudo de espalhamento Raman nos ácidos palmítico e esteárico: forma C [tese]. Fortaleza (CE): Universidade Federal do Ceará; 2010.

3. Silva EL. Desenvolvimento de nanopartículas lipídicas sólidas contendo ácido retinóico e ácido láurico para o tratamento tópico da acne vulgaris [dissertação]. Belo Horizonte (MG): Universidade Federal de Minas Gerais; 2011

4. Silva WC. Produção enzimática de biodiesel a partir de óleos láuricos em reatores de leito fixo duplo estágio incorporando coluna extratora do glicerol formado como subproduto [dissertação]. Lorena (SP): Universidade de São Paulo; 2013.

5. Prajapati P, Prajapati A. Raman Spectroscopy: A versatile tool in pharmaceutical analysis. Int. J. Pharm. Sci. Rev. Res. 2011; 9: 57-64.

6. Moreno E, Cordobilla R, Calvet T, Cuervas-Diarte MA, Cuevas-Diarte MA, Gbabode G, Negrier P, Mondieig D, Oonk HAJ. Polymorphism of even saturated carboxylic acids from n-decanoic to neicosanoic acid. New Journal of Chemistry. 2007 apr; 31: 947-957. doi: 10.1039/b700551b.

7. Vogel-Weill C, Gruger, A. Etude de la conformation des acides n-nonanoique, Z et E-9 octadecenoiques à $90 \mathrm{~K}$ par spectrométries infrarouge et Raman II - Etude de la conformation des chaines hydrocarbonées des acides $\mathrm{Z}$ et E-9 octadecenoiques à $90 \mathrm{~K}$ par spectrométrie infrarouge et Raman. Spectrochimica Acta Part A: Molecular and Biomolecular Spectroscopy. 1996 apr; 52: 1737 1755.

8. De Gelder J, Gussem K, Vandenabeele P, Moens L. Reference database of Raman spectra of biological molecules. J . Raman Spectrosc. 2007; 38: 1133-1147. doi: 10.1002/jrs.1734.

9. Saraiva GD, Nogueira CES, Freire PTC, Sousa FF, Silva JH, Teixeira AMR,

Mendes Filho J. Temperature-dependent vibrational spectroscopic study and DFT calculations of the sorbic acid. Spectrochimica Acta Part A: Molecular and Biomolecular Spectroscopy. 2015; 137: 14091416. doi: 10.1016/j.saa.2014.08.142.

10. Sousa FF, Freire PTC, Menezes AS, Pinheiro GS, Cardoso LP, Alcantara Jr. P, Moreira SGC, Melo FEA, Mendes Filho J, Saraiva GD. Low-temperature phase transformation studies in the stearic acid: C form. Spectrochimica Acta Part A: Molecular and Biomolecular Spectroscopy. 2015 Apr; 148: 80-288, doi: 10.1016/j.saa.2015.04.003.

11. Vogel-Weill C, Corset J. Espectres infrarouge et Raman de l'acide stéarique et d'une série d'acides gras forme C: modes de squellete, couplage des modes longitudinaux acoustiques (LAM1,LAM3) avec les modes dans le plan de la liaison hydrogéne du dimére en dessous de $700 \mathrm{~cm}-1$. Spectrochimica Acta Part A. 1995 apr; 51: 2357-2377. 